hep-ph/9412301

RAL-94-122

OUTP-94-35P

\title{
The photoproduction of hybrid mesons from CEBAF to HERA
}

\author{
Frank E. Close* \\ Particle Theory, Rutherford-Appleton Laboratory, Chilton, Didcot OX11 0QX, UK \\ Philip R. Page ${ }^{\dagger}$ \\ Theoretical Physics, University of Oxford, 1 Keble Road, Oxford OX1 3NP, UK
}

December 1994

\begin{abstract}
Decay widths, branching ratios and production dynamics of some recently discovered $J^{P C}=1^{-+}, 0^{-+}, 1^{--}$and $2^{-+}$mesons are found to be in remarkable agreement with the predicted properties of hybrid mesons. We propose tests for this new dynamics, emphasise the critical role of $\pi b_{1}$ or $\pi h_{1}$ decay channels in discriminating hybrids from conventional states, and suggest that photoproduction may offer special opportunities for isolation and confirmation of hybrids.
\end{abstract}

\footnotetext{
*E-mail : fec@v2.rl.ac.uk

${ }^{\dagger}$ E-mail : p.page@physics.oxford.ac.uk
} 
There is a substantial area in the Standard Model of particles and interactions where we remain fundamentally ignorant: while the gluon degrees of freedom expressed in the Lagrangian of Quantum Chromodynamics have been established beyond doubt in high momentum data, their dynamics in the strongly interacting limit epitomised by hadron spectroscopy are quite obscure. It is possible that this is about to change [1]].

For the first time there is a candidate scalar glueball [2, 3, 4] whose mass $1.5 \sim 1.6 \mathrm{GeV}$ is consistent with the prediction of $1550 \pm 50 \mathrm{MeV}$ from lattice studies of QCD [5]. Furthermore, its production and decay properties in a range of experiments fit more naturally with those of a gluonic excitation than of a conventional quark - antiquark $(q \bar{q})$ nonet state. If these are the first hints of gluonic excitation at a mass scale anticipated by theory, then this raises the possibility that in the $1.5-2 \mathrm{GeV}$ mass range there is a rich spectroscopy of "hybrid" states where the gluonic fields or "flux-tubes" are excited in the presence of coloured quark sources [6, 7, 8]. In this letter we present evidence supporting this notion and propose ways of testing the hypothesis in forthcoming experiments.

It is well known that hybrid mesons can have quantum numbers for spin parity and charge conjugation $\left(J^{P C}\right)$ in combinations such as $0^{+-}, 1^{-+}, 2^{+-}$etc. which are unavailable to conventional mesons and as such provide a potentially sharp signature for hybrids. A central theme in this letter will be to note that even when hybrid and conventional mesons have the same $J^{P C}$ quantum numbers, they may still be distinguished. The essential reason is that although superficially identical in their overall quantum numbers, the two states have different internal structures which give rise to characteristic selection rules [8, 9]. Decays into $\pi b_{1}$ or $\pi h_{1}$ are pivotal here: we illustrate this for the particular case of $1^{--}$ states [9] such as may be produced in $e^{+} e^{-}$annihilation or diffractive photoproduction.

Specifically:

(i) If $q \bar{q}$ in either hybrid or conventional mesons are in a net spin singlet configuration then the dynamics of the flux-tube forbids decay into final states consisting only of spin singlet mesons.

For $J^{P C}=1^{--}$states this selection rule distinguishes between conventional vector mesons which are ${ }^{3} S_{1}$ or ${ }^{3} D_{1}$ states and hybrid vector mesons where the $q \bar{q}$ are coupled to a spin singlet. This implies that in the decays of hybrid $\rho$, the channel $\pi h_{1}$ is forbidden whereas $\pi a_{1}$ is allowed and that $\pi b_{1}$ is analogously suppressed for hybrid $\omega$ decays; this is quite opposite to the case of ${ }^{3} L_{1}$ conventional mesons where the $\pi a_{1}$ channel is relatively suppressed and $\pi h_{1}$ or $\pi b_{1}$ are allowed [10, 11]. The extensive analysis of data in ref. [12] revealed the clear presence of $\rho(1460)$ 13] with a strong $\pi a_{1}$ mode but no sign of $\pi h_{1}$, in accord with the hybrid situation. Furthermore, ref. 112 finds evidence for $\omega(1440)$ with no visible decays into $\pi b_{1}$ which again is in significant contrast to the expectations for conventional $q \bar{q}\left({ }^{3} S_{1}\right.$ or $\left.{ }^{3} D_{1}\right)$ initial states and in accord with the hybrid configuration.

(ii) The dynamics of the excited flux-tube in the hybrid state suppresses the decay to mesons where the $q \bar{q}$ are ${ }^{3} S_{1}$ or ${ }^{1} S_{0}$ " $L=0$ " states. The preferred decay channels are to $(L=0)+(L=1)$ pairs [8, 14]. Thus in the decays of hybrid $\rho \rightarrow 4 \pi$ the $\pi a_{1}$ content is predicted to be dominant and the $\rho \rho$ to be absent. The analysis of ref. [12] finds such a pattern for $\rho(1460)$.

(iii) The selection rule forbidding $(L=0)+(L=0)$ final states no longer operates if the internal structure or size of the two $(L=0)$ states differ [8, [15]. Thus, for example, decays to $\pi+\rho, \pi+\omega$ or $K+K^{*}$ may be significant in some cases [9]. Though still suppressed relative to the dominant pathway, (ii) above, this too is the case for $\rho(1460)$. It is also interesting to note that for a hybrid $\omega(1440)$, the preferred $(L=0)+(L=1)$ decay paths 
are predicted to be kinematically suppressed leaving the $\pi \rho$ and possibly $\eta \omega$ as dominant decays 9 .

(iv) In the case of production, where an exchanged $\pi, \rho$ or $\omega$ is involved, it is possible that the strength could be significant at least to the extent that the exchanged off massshell state may have different structure to the incident on-shell beam particle 16. This may be particularly pronounced in the case of photoproduction where couplings to $\rho \omega$ or $\rho \pi$, which were suppressed in ref.[9], could be considerable when the $\rho$ is effectively replaced by a photon and the $\omega$ or $\pi$ is exchanged. As we shall discuss later, this may explain the production of the candidate exotic $J^{P C}=1^{-+}$(ref.[17]) and a variety of anomalous signals in photoproduction.

According to the flux-tube model, the $J^{P C}$ quantum numbers of the lowest lying hybrid mesons may be divided into two classes :

(a) $0^{-+}, 1^{+-}, 1^{--}, 1^{++}, 2^{-+}$(deemed "conventional" in that they can also be shared by standard $q \bar{q}$ states), and

(b) $0^{+-}, 1^{-+}, 2^{+-}$(deemed "exotic").

In the hybrid $1^{--}$and $1^{++}$the $q \bar{q}$ are coupled to a spin singlet; all other $J^{P C}$ are spin triplets.

If the $\rho(1460)$ has signposted the existence of the vector hybrid nonet, then we need to establish which of the other seven multiplets should also be visible. States whose couplings are predicted to be strong, with highly visible decay channels and moderate widths relative to the $\rho$ candidate, must be seen if hybrids are to be established. Conversely, channels where no signals are seen should be those whose signals are predicted in refs. [8, 9] to be weak. We shall make the case that these criteria do appear to be realised in the data.

$\underline{\operatorname{Exotic} J^{P C}}$

The most obvious signature for a hybrid meson is the appearance of a flavoured state with an exotic combination for $J^{P C}$. It was noted in ref. [14] and confirmed in ref. [9] that the $0^{+-}$width is predicted to be over $1500 \mathrm{MeV}$ thereby rendering the state effectively invisible. The $2^{+-}$is also predicted to be very broad and hard to see if its mass is $\geq 1.9 \mathrm{GeV}$. The best opportunity for isolating exotic hybrids appears to be in the $1^{-+}$wave where, for the $\mathrm{I}=1$ state with mass around $2 \mathrm{GeV}$, partial widths are typically

$$
\pi b_{1}: \pi f_{1}: \pi \rho=170 \mathrm{MeV}: 60 \mathrm{MeV}: 10 \mathrm{MeV}
$$

The narrow $f_{1}(1285)$ provides a useful tag for the $1^{-+} \rightarrow \pi f_{1}$ and ref. 17 has recently reported a signal in $\pi^{-} p \rightarrow\left(\pi f_{1}\right) p$ at around $2.0 \mathrm{GeV}$ that appears to have a resonant phase though they admit that more data are required for a firm conclusion.

The partial widths for $\pi b_{1}$ and $\pi f_{1}$ in eqn.(1) are similar to those predicted in ref. [14] but we note also the possibility that the $\pi \rho$ channel is not negligible relative to the signal channel $\pi f_{1}$ [9]. This may be important in view of the puzzle, commented upon in ref. [17], that significant $\pi+\rho$ coupling may be present in the production mechanism. In view of our analysis in ref. [9] and property (iv) above, it is clear that the latter coupling may be significant in $\pi p \rightarrow 1^{-+} p$. We shall comment on the possible photoproduction of this state later.

Conventional $J^{P C}$

Previous discussions of the dynamics of hybrids in flux-tube models have been limited to exotic $J^{P C}$ [14; the first calculation of the widths and branching ratios of hybrid mesons 
with conventional quantum numbers is in ref. [9]. This predicts that for hybrids at $\sim 2 \mathrm{GeV}$ made from $u, d$ flavoured quarks the $1^{+-}, 1^{++}$states are over $500 \mathrm{MeV}$ wide in both $\mathrm{I}=0,1$ states; by contrast the $0^{-+}, 2^{-+}$and the $1^{--}$are predicted to be potentially accessible. Hence we predict that the most visible $J^{P C}$ states are those that coincide with the lowest lying hybrids of the bag model, namely $1^{--} ;(0,1,2)^{-+}$[6, 7]. Moreover, the $q \bar{q}$ spin content is identical in the two models. It is therefore interesting that each of these $J^{P C}$ combinations shows rather clear signals with features characteristic of hybrid dynamics and which do not fit naturally into a tidy $q \bar{q}$ conventional classification.

We have already mentioned the $1^{--}$which motivated this analysis: a more detailed discussion may be found in ref. [9].

Turning to the $0^{-+}$wave, we note that the VES Collaboration at Protvino sees an enigmatic and clear $0^{-+}$signal in diffractive production with $37 \mathrm{GeV}$ incident pions on beryllium [18]. They study the channels $\pi^{-} N \rightarrow \pi^{-} \pi^{+} \pi^{-} N ; \pi^{-} K^{+} K^{-} N$ and see a resonant signal $M \approx 1790 \mathrm{MeV}, \Gamma \approx 200 \mathrm{MeV}$ in the $(L=0)+(L=1) \bar{q} q$ channels $\pi^{-}+f_{0} ; K^{-}+$ $K_{0}^{*}, K(K \pi)_{S}$ with no corresponding strong signal in the kinematically allowed $L=0$ two body channels $\pi+\rho ; K+K^{*}$ :

$$
\begin{aligned}
& \frac{0^{-+} \rightarrow \pi^{-} \rho^{0}}{0^{-+} \rightarrow \pi^{-} f_{0}(1300)}<0.3(95 \% \text { C.L. }) \\
& \frac{0^{-+} \rightarrow K^{-} K^{*}}{0^{-+} \rightarrow\left(K^{-} K^{+} \pi\right)_{S}}<0.1(95 \% \text { C.L. })
\end{aligned}
$$

The width and large couplings to kaons are both surprising if this were the second radial excitation of the pion (the first radial excitation is seen as a broad enhancement in accord with expectations). Furthermore, the preference for decay into $(L=0)+(L=1)$ mesons at the expense of $L=0$ pairs is in accord with expectations for hybrids.

The resonance also appears to couple strongly to the enigmatic $f_{0}(980)$ :

$$
\frac{0^{-+} \rightarrow \pi^{-} f_{0}(980)}{0^{-+} \rightarrow \pi^{-} f_{0}(1300)}=0.9 \pm 0.1
$$

As noted in ref. [18] this is an unexpectedly high value since the $f_{0}(980)$ has a small width and strong coupling to strangeness while the $f_{0}(1300)$ is a broad object coupled mainly to non-strange quarks. However, this may be natural for a hybrid at this mass for the following reason. The $K K_{0}^{*}$ channel would be dominant (table 3 of ref. [9]) but for the fact that it is kinematically suppressed; however, the $\sim 300 \mathrm{MeV}$ width of the $K_{0}^{*}(1430)$ will feed the $(K K \pi)_{S}$ (as observed [18]) and help to feed the channel $\pi f_{0}(980)$ through the strong affinity of $K \bar{K} \rightarrow f_{0}(980)$. Thus the overall expectations for hybrid $0^{-+}$are in line with the data of ref. 18. Important tests are now that there should be a measureable decay to the $\pi \rho$ channel with only a small $\pi f_{2}$ or $K K^{*}$ branching ratio.

This leaves us with the $2^{-+}$.

There are clear signals of unexplained activity in the $2^{-+}$wave in several experiments for which a hybrid interpretation may offer advantages.

Historically the ACCMOR Collaboration [19] noted a $2^{-+}$structure around $1.8 \mathrm{GeV}$, coupled to $\pi f_{2}$, and too near to the $\pi_{2}(1670)$ for these to be the $1^{1} D_{2}$ and $2^{1} D_{2}$ (radial excitation) of conventional quarkonium. Chanowitz and Sharpe suggested [7] that the 1.8 $\mathrm{GeV}$ state might set the mass scale for hybrid excitations. This structure is tantalisingly 
similar to sightings of a possible $2^{-+}$(or even $1^{-+}$, see below) at $1.77 \mathrm{GeV}$, width $100-200$ $\mathrm{MeV}$ in photoproduction via $\pi$ exchange [20] and coupled to $\pi \rho$ and $\pi f_{2}$. An earlier low energy photoproduction experiment [21] also shows a clear structure at 1.7 - 1.9 GeV though its quantum numbers are not identified; we note that hybrid $2^{-+}, 1^{-+}$are both favourably photoproduced according to the table 7,8 in ref. [9] via $\pi$ exchange or $\omega$ exchange. There are also some indications of a doubling of states in the $I=0 \eta \pi^{0} \pi^{0}$ channel where the Crystal Barrel at LEAR 22 finds both $\eta_{2}(1650)$ (which is probably the partner of $\pi_{2}(1670)$ ) and also a candidate $\eta_{2}(1850)$ decaying into $f_{2} \eta$ (unlikely to be $s \bar{s}$ ).

If these various experiments are heralding activity in the $2^{-+}$wave, a search for the $\pi b_{1}$ decay channel [9] becomes pivotal. This follows once again from the selection rule forbidding the decay of a spin singlet meson into pairs of spin singlets: this prevents the decay of ${ }^{1} D_{2}\left(\pi_{2}\right) \rightarrow b_{1} \pi$ whereas this channel is allowed and potentially significant for $\pi_{2}^{\text {hybrid }}[9]$.

In this context, the results of ref. 23 are interesting. They studied $\gamma p \rightarrow\left(b_{1} \pi\right) p$ at 25-50 GeV incident energy with the specific intention of seeking hybrids. At that time the only calculations of hybrid branching ratios in the literature were for the exotic $J^{P C}$ states and ref. 23] assumed them to be a guide to the non-exotic cases also. Our calculations 99 show that this assumption is not generally true. In particular, as noted above, the selection rule forbids the $b_{1} \pi$ decay modes for the case of hybrid $\omega$ and conventional ${ }^{1} D_{2}$ $\left(\pi_{2}\right)$ quarkonium while allowing it for conventional $\left({ }^{3} S_{1}\right.$ or $\left.{ }^{3} D_{1}\right) \omega$ or hybrid $\pi_{2}$ respectively. As the $b_{1} \pi$ is the trigger channel in ref. 23] several of the conclusions of that experiment merit re-examination. In particular, if one insists on diffractive production then any signals are not hybrid $1^{--}$; conversely, if one allows also $\omega$ exchange (which is not negligible at $25-50 \mathrm{GeV}$ ), then either (or both!) hybrid $2^{-+}, 1^{-+}$production can feed the $b_{1} \pi$ signal but conventional ${ }^{1} D_{2}$ quarkonium is forbidden in this mode. $\omega$ exchange also can feed $1^{++}$: a $2^{3} P_{1} \rightarrow b_{1} \pi$ is allowed whereas a hybrid $1^{++}$is forbidden in this channel.

These various signals in the desired channels provide a potentially consistent picture. The challenge now is to test it. Dedicated high statistics experiments with the power of modern detection and analysis should re-examine these channels. As several of these candidates appear in photoproduction, it may be worthwhile to consider the possibility that the $(L=0)+(L=0)$ suppression (into $\rho \omega$ or $\rho \pi)$ may be overruled when the " $\rho$ " is a $\gamma$. With this in mind we identify from tables 7-9 of ref. [9] the potentially significant photon couplings (where "photon" is equated with $\rho$ via vector meson dominance and the exchanged particle is $\omega$ or $\pi$ in this analysis).

The unsuppressed widths are for $\mathrm{I}=1$ hybrid states (in $\mathrm{keV}$ )

$$
\begin{aligned}
& 2^{-+} \rightarrow \pi^{+} \gamma=70 ; \quad \omega \gamma=250 \\
& 1^{-+} \rightarrow \pi^{+} \gamma=70 ; \quad \omega \gamma=180 \\
& 0^{-+} \rightarrow \pi^{+} \gamma=275 ; \quad \omega \gamma=0 \\
& 1^{++} \rightarrow \pi^{+} \gamma=145 ; \quad \omega \gamma=455
\end{aligned}
$$

and for the $I=0$ hybrid sector important widths include

$$
\begin{aligned}
& 2^{-+} \rightarrow \rho \gamma= \\
& 1^{-+} \rightarrow \rho \gamma=90 \\
& 1^{++} \rightarrow \rho \gamma=220
\end{aligned}
$$




$$
1^{--} \rightarrow \pi \gamma=120
$$

The $1^{++}$channel has the strongest photon couplings but is predicted to be broad and hard to isolate (unless its mass is unexpectedly low, as for the enigmatic $f_{1}(1430)$ in the isoscalar sector). Both $1^{-+}, 2^{-+}$have similar photoproduction rates and healthy $b_{1} \pi$ branching ratios and hence are candidates for the signal in ref. 23. The $0^{-+}$may be prominent in low energy photoproduction where $\pi$ exchange is important but its $\omega \gamma$ coupling is suppressed by a selection rule for hybrids which will disfavour the $0^{-+}$photoproduction at higher energies.

The above are all upper limits on the electromagnetic couplings and may be used to estimate upper limits for the photoproduction rates. In the case of $\pi$ exchange, at least, there is reason to expect that the actual strengths will be $\geq 20 \%$ of these [9]. This is especially favourable to low-energy photoproduction and as such offers a rich opportunity for the programme at an upgraded CEBAF or possibly even at HERA. If the results of ref. 23] are a guide, then photoproduction may be an important gateway at a range of energies and the channel $\gamma+N \rightarrow\left(b_{1} \pi\right)+N$ can discriminate hybrid $1^{--}$and $2^{-+}$from their conventional counterparts.

We also note that in $\pi^{-} p \rightarrow\left(f_{1} \pi^{-}\right) p$ ref. [17] sees a broad $1^{++}$signal that is more prominent than their exotic $1^{-+}$candidate. They interpret this $1^{++}$as a radial excitation; however, we note that if the production mechanism involves $\rho$ exchange, then the hybrid couplings predicted in ref. [9] imply that hybrid $1^{++}$should be produced in this same experiment with a greater strength than the $1^{-+}$. Experimentally it may be possible to distinguish between the radial ${ }^{3} P_{1}$ axial meson and the hybrid (spin singlet) configuration by studying $\pi N$ or $\gamma N \rightarrow\left(b_{1} \pi\right) N$ : a hybrid $1^{++}$decouples from this channel.

Thus to summarise, we suggest that data are consistent with the existence of low lying multiplets of hybrid mesons based on the mass spectroscopic predictions of ref. [8] and the production and decay dynamics of ref. [9]. Specifically the data include

$$
\begin{aligned}
& 0^{-+}(1790 \mathrm{MeV} ; \Gamma=200 \mathrm{MeV}) \rightarrow \pi f_{0} ; K \bar{K} \pi \\
& 1^{-+} \quad(\sim 2 \mathrm{GeV} ; \Gamma \sim 300 \mathrm{MeV}) \rightarrow \pi f_{1} ; \pi b_{1}(?) \\
& 2^{-+}(\sim 1.8 \mathrm{GeV} ; \Gamma \sim 200 \mathrm{MeV}) \rightarrow \pi b_{1} ; \pi f_{2} \\
& 1^{--}(1460 \mathrm{MeV} ; \Gamma \sim 300 \mathrm{MeV}) \rightarrow \pi a_{1}
\end{aligned}
$$

Detailed studies of these and other relevant channels are called for together with analogous searches for their hybrid charmonium analogues, especially in photoproduction or $e^{+} e^{-}$annihilation.

\section{Acknowledgements}

We thank T. Barnes, A. Donnachie and several experimental colleagues at the Rutherford Appleton Laboratory for helpful discussions.

\section{References}

[1] C. Amsler, in Proceedings of $27^{\text {th }}$ International Conf. on High Energy Physics, Glasgow, July 1994 (unpublished); F.E.Close, ibid.; F.E.Close and C.Amsler, (unpublished). 
[2] V. Anisovich et al., Phys. Lett. B323 233 (1994)

[3] A. Kirk, (NA12/2 Collaboration), CERN Report No. CERN/SPSLC 94-22, p. 281.

[4] D.V. Bugg, in Proceedings of International Symposium on Medium Energy Physics, Beijing, Aug. 1994 (unpublished).

[5] G. Bali et al. (UKQCD), Phys. Lett. B309 378 (1993).

[6] T. Barnes and F.E. Close, Phys. Lett. B116 365 (1982).

T. Barnes, F.E. Close, F. de Viron, Nucl. Phys. B224 241 (1983).

[7] M. Chanowitz, S. Sharpe, Nucl. Phys. B222 211 (1983).

[8] N. Isgur, J. Paton, Phys. Rev. D31 2910 (1985).

[9] F.E. Close and P. Page, Rutherford Appleton Laboratory, Report No. RAL-94-116; OUTP-94-29P; hep-ph/9411301 (unpublished).

[10] G. Busetto and L. Oliver, Z. Phys. C20 247 (1983).

[11] R. Kokoski, N. Isgur, Phys. Rev. D35 907 (1987).

[12] A.B. Clegg and A. Donnachie, Z. Phys. C62 455 (1994)

[13] Particle Data Group, Phys. Rev. D50 1173 (1994)

[14] N. Isgur, R. Kokoski and J. Paton, Phys. Rev. Lett. 54869 (1985).

[15] M. Tanimoto et al. Phys. Lett. 116B 198 (1982)

A. Le Yaouanc et al. Z.Phys C28 309 (1985)

F. Iddir et al. Phys. Lett. B205 564 (1988)

[16] A. Donnachie (private communication).

[17] J.H. Lee et al., Phys. Lett. B323 227 (1994)

[18] A. Zaitsev (VES Collaboration), in Proceedings of $27^{\text {th }}$ International Conf. on High Energy Physics, Glasgow, July 1994 (unpublished).

[19] C. Daum et al. (ACCMOR Collaboration), Nucl. Phys. B182 269 (1981)

[20] D. Aston et al., Nucl. Phys. B189 15 (1981)

G. Condo et al., Phys. Rev. D43 2787 (1991).

[21] Y. Eisenberg et al. Phys. Rev. Lett. 231322 (1969).

[22] A. Cooper, Ph.D. thesis, Queen Mary and Westfield College, London University, 1994 (unpublished).

[23] M.Atkinson et al. Z.Phys. C34 157 (1987) 